\title{
VII.
}

\section{La separazione dello Stato dalla Chiesa nel Paese di Galles}

\author{
del \\ Prof. Dr. Andrea Galante
}

(Innsbruck).

\begin{abstract}
Allorquando, durante le elezioni generali inglesi del 1885, veniva risollevata in Inghilterra la questione della separazione dello Stato della Chiesa il Gladstone veniva interpellato dal Döllinger sullo stato della questione e sulla probabilità 0 meno che potesse venir risolta. ${ }^{1}$ ) Nella sua risposta il Gladstone distingueva fra l'aspetto generale della questione e le circostanze particolari di essa nelle diverse parti del Regno Unito. ${ }^{2}$ ) Egli riteneva che per l'Inghilterra propriamente detta, quand'anche vi fosse una maggioranza favorevole alla separazione, questa non avrebbe potuto venir attuata per lungo tempo, invece per la Scozia era d'arviso che la questione avesse essenzialmente un carattere locale e che la separazione fosse possibile ove il popolo scozzese lo desiderasse. Per riguardo al Paese di Galles il Gladstone riconosceva che la Chiesa di Stato Anglicana vi costituiva una minoranza, peraltro per il fatto che essa era storicamente ed organicamente congiunta alla Chiesa anglicana generale credeva che il separare lo Stato dalla Chiesa nel Paese di Galles dovesse andar congiunto a gravi difficoltà. Le previsioni del Gladstone sui diversi aspetti delle relazioni fra lo Stato e la Chiesa, a seconda delle diverse circostanze

1) Cf. Galante, Lo Stato e la Chiesa nella mente e nell' opera politica di W. E. Gladstone, nella Festschrift zum 70. Geburtstag Otto Gierkes. Weimar 1911. p. 938. - 2) Vedasi la lettera del Gladstoue in D. C. Lathbury, Correspondence on Church and Religion W. E. Gladstone. London 1910. I. p. $183 \mathrm{seg}$.
\end{abstract}


locali e regionali della Granbrettagna, venivano confermate dal successivo svolgimento della politica ecclesiastica inglese. Le vicende politiche degli ultimi decennii venivano mettendo sempre più in risalto la questione della separazione nel Paese di Galles ed ora il Parlamento inglese sarà chiamato a decidersi sul Welsh Church Bill, presentato dal Ministero Asquith alla Camera dei Comuni, che mira appunto a togliere alla Chiesa anglicana (Established Church of England) il carattere di Chiesa di Stato nelle contee del Paese di Galles. Ora le questioni ecclesiastiche gallesi sono cosi intimamente congiunte alle condizioni geografiche, etnografiche e storiche, che prima di esaminare il Welsh Church Bill é necessario, per ben comprenderne la portata, di gettare uno sguardo retrospettivo alle peculiari circostanze del Paese di Galles.

Il Paese di Galles (Wales) é costituito dalla grande penisola situata a sud-ovest dell' Inghilterra ${ }^{1}$ ), delimitata a settentrione dal Mare Irlandese e dall' estuario del fiume Dee, ad occidente dal Canale di S. Giorgio, a mezzogionno dal Canale di Bristol, mentre ad oriente confina colle contee di Cheshire, Shropshire, Herefordshire e Montmouthshire secondo la delimitazione stabilita nel sec. XVI da una legge di Enrico VIII (27. Henry VIII, c. 26). Esso é costituito da tredici contee e cioè : Anglesey, Carnarvonshire, Denbighshire, Flintshire, Merionethshire, Montgomeryshire, Brecknockshire, Cardiganshire, Carmarthenshire, Glamorganshire, Pembrokeshire, Radnorshire, Monmouthshire. Le prime sei contee costituiscono il Paese di Galles settentrionale (North Wales), le altre il Paese di Galles meridionale (South Wales). Il Monmouthshire é propriamente una contea inglese', e come tale veniva nella citata legge di Enrico VIII compresa nella giurisdizione delle Corti di Westminster, ma in seguito, sotto l'aspetto amministrativo e legislativo, venne generalmente considerato come parte integrante del Paese di Galles. Secondo il censimento del 1901 la sua popolazione era di

1) Cf. Carlisle, Topography of Wales. London 1811; e J. RhysD. Brynmor Jones, The Welsh People. Chapters on their Origin, History, Laws, Language, Literature and Characteristics, 2. ed. London 1900, p. IX seg. 
2.012.876 abitanti di fronte a 41.976.827 abitanti del Regno unito.

Il Paese di Galles é essenzialmente costituito da montagne separate fra loro da profonde valli; più della metà del suo territorio é situata ad un altezza di oltre $170 \mathrm{~m}$. sul livello del mare, e le sue catene di monti hanno avuta una grande importanza sulla sua evoluzione storica, come sulla formazione del carattere dei suoi abitanti. La montagna, come fu giustamente osservato ${ }^{1}$ ) per una parte diede agli abitanti le loro particolari caratteristiche in contrasto coi paesi circostanti, difendendoli dai nemici esterni, e al tempo stesso la divisione in valli rese difficile la unificazione politica del suo territorio. L'elemento fondamentale della popolazione del Paese di Galles ${ }^{2}$ ) é di razza celtica, affine, etnograficamente, a quello dell' Irlanda, dell' isola di Man, della Scozia e dell' Armorico. La popolazione primitiva é costituita dai cosidetti Tverni, comprendendosi sotto questa denominazione gli abitanti del periodo pre-celtico, di cui si conservano traccie nella popolazione attuale. Ad essi si aggiunse in seguito l'elemento celtico rappresentato dai Bretoni e Goideli, diverso per la costituzione fisica, per la organizzazione sociale e probabilmente per le istituzioni religiose. Lo sviluppo storico del Paese di Galles fu profondamente modificato dalla occupazione romana, che fu la conquista più occidentale dei Romani, compiutasi nella seconda metà del I secolo dell' era volgare, malgrado la tenace resistenza dell' elemento celtico. Per essa non solo la civiltà romana si estendeva al Paese di Galles, ma veniva compiuta una larga opera di latinizzazione dei suoi abitanti. ${ }^{3}$ )

1) O. M. Edwards, Wales, 4. ed. London 1907 (nelle serie The Story of the Nations) cap. I. - ${ }^{2}$ ) Cf. Rhys, Celtic Britain. London 1884, 2. ed.; Rhys-Brynmor Jones, The Welsh People, cit., p. 1-35. 3) Per la occupazione romana del Paese di Galles cf. Rhys-Brynnor Jones, o. c. p. 75-115 colla letteratura ivi citata, vedasi anche Poole, Historical Atlas of modern Europe from the Decline of the Roman Empire Oxford 1896. tav. XV. - Per la storia generale del Paese di Galles ef. J. William, History of Wales, 1869; J. Evans, A popular History of the Ancient Britons or the Welsh People, London 1901; Edwards, Wales, cit. e specialmente ora l'opera fondamentale di J. E. Lloyd, A History of Wales from the earliest Times to the Edwardian Conquest. London 1911. 2 vol. 
Dopo che al principio del $\mathrm{V}$ secolo furono ritirate le legioni romane, al Dux Brittaniarum succedette il Gwedlig, che dominò le tribù bretoni, goideliche ed iverne, in cui l'elemento bretone divenne predominante. Esse furono designate col nome comune di Combroyes o Cymry, mentre il nome di Wales (da Weleas = stranieri) fu dato poi agli indigeni dai conquistatori anglo-sassoni.

In questo regno cimrico fermatosi dopo la partenza dei Romani si mantiene l'unità politica del Paese di Galles e nella leggenda di $\operatorname{Re}$ Arturo é consacrato il campione mitico della sua tradizionale unità. ${ }^{1}$ )

L'opera dei re del Paese di Galles é essenzialmente rivolta in quest' epoca a mantenere la continuità dell'unione politica di fronte alla ribellione continua dei principi, agli attacchi inglesi dalla parte di terra ed a quelli danesi per la via del mare. Al principio del sec. XI l'invasione danese getta il paese in uno stato di completa anarchia e pochi decennii dopo il Paese di Galles si leva contro la conquista normanna, che si venne compiendo solo gradatamente e con grandi lotte.

All' epoca in cui in Inghilterra si combatte la grande lotta fra i baroni del Regno e il Re Giovanni, che doveva condurre alla concessione della Magna Charta, l'ideale del Paese di Galles si compendia nell' opera di Llywelyn il Grande (1174-1240), che mira a consolidarlo come unità politica, compresa bensi nel sistema feudale inglese, ma distinta per lingua, per razza e per territorio.

I successori di Llywelyn divisi e in lotta fra di loro non riescono a mantenere l'indipendenza del Paese di Galles e dopo la morte di Enrico III si combatte l'ultima lotta per l'indipendenza del Paese di Galles, che termina verso il 1284 colla piena conquista del suo territorio per parte di Edoardo I. Così si compiva l'annessione del Paese di Galles alla corona inglese, l'amministrazione veniva riorganizzata sulla base del sistema feudale inglese, pur mantenendosi tuttavia l'osservanza dell' antico diritto gallese. ${ }^{2}$ ) Nel 1301 il Principe ereditario

1) Cf. Rhys, Studies in the Arthurian Legend. 1891. - ${ }^{2}$ ) Per l'antico diritto del Paese di Galles ef. Ancient Laws and Institutes of Wales. London 1841 (pubblicato nella raccolta ufficiale dei Commissioners 
Edoardo, che era nato a Carnarvon e che nel 1307 saliva al trono col titolo di Edoardo II. veniva creato Principe di Galles e tale dignità rimaneva fino all' epoca odierna il titolo tradizionale dell' erede al trono d'Inghilterra.

Al principio del sec. XV Owen Glendower dell' antica famiglia dei principi di Powys si pone a capo di una rivolta intesa a restaurare l'indipendenza del Paese di Galles, ma senza effetto. L'accessione dei Tudor di origine gallese segna la fusione del Paese di Galles coll' Inghilterra. Essa diede luogo ad una grande e profonda riorganizzazione politica, congiunta con un' ampia riforma giudiziaria, mentre al tempo stesso veniva introdotta la Riforma anglicana nelle contee gallesi.

Colle legge di Enrico VIII del 1535 (27. Henry VIII c. 26) il Paese di Galles veniva definitivamente riunito all' Inghilterra e se per una parte segnava l'abolizione della lingua gallese, come lingua ufficiale, e delle sue peculiarità legislative e consuetudinarie, dall' altra accordava al Paese di Galles una rappresentanza in Parlamento, mantenendo la sua organizzazione giudiziaria separata, con a capo le Great Sessions. Solamente nel 1830 queste venivano abolite, e con esse scompariva un' ultima traccia dell'autonomia politica del Paese di Galles. Ma non ostante che in tal modo fosse compiuta la fusione amministrativa delle contee gallesi con quelle inglesi, il Parlamento inglese accordava in seguito, per alcune particolari materie, alcune leggi speciali per il Paese di Galles, come il Sunday closing Act del 1881 e il Welsh intermediate Education Act del 1889.

Compiuta la fusione legale e amministrativa dell'Inghilterra col Paese di Galles, questo conservò tuttavia la propria lingua che rimase come simbolo della sua autonomia nazionale. La lingua gallese $\left(W^{\top} \text { elsh }\right)^{1}$ ) consacrata da insigni opere poetiche medioevali, raggiunge nel sec. XII e XIII un alto valore letterario. ${ }^{2}$ ) Dopo l'incorporazione del Paese

of Public Records): A. W. Wade-Evans, Welsh Medieval Law. London 1909; F. Se ebohm, The Welsh tribal System, 1895; Rhys-Brynmor Jones, o. c. cap. VI. p. 176-270, cap. VIII-IX. p. 346-452.

1) Cf. Zeu ss, Gramatica celtica, 2. ed. di Hebel, 1871; J. MorrisJones, Historical Welsh Grammar, I. 1911. - ${ }^{2}$ ) Cf. T. Stephens, Literature of the kymry during the XII and two succeeding Centuries, 2. ed. 1876. 
di Galles all' Inghilterra nel 1535, essa parve minacciata, in quanto le classi alte abbandonarono l'uso della lingua gallese ritenuto come segno di inferiorità e di soggezzione ${ }^{1}$ ), ma poco dopo l'introduzione della Riforma il Book of Common Prayer e quindi il Nuovo Testamento e poi l'intera Bibbia venivano tradotti in gallese, e ciò contribuiva a mantener vivo l'uso della lingua nazionale nel popolo. Ma il moderno risveglio della lingua e letteratura gallese, per cui l'elemento religioso viene ad unirsi al moto nazionale, si ricollega alla istituzione di scuole domenicali (Sunday Schools) nel Paese di Galles, iniziata da Thomas Charles ${ }^{2}$ ), che divennero il centro popolare dell' insegnamento della lingua gallese. Negli ultimi tempi venne ravvivata l'antica consuetudine dell' Eisteddfod, così caratteristica del Paese di Galles, e che consiste in una grande adunanza nazionale con gare di poesia, prosa, musica vocale e istrumentale, e che ebbe una grandissima importanza nella restaurazione della letteratura e dell' arte gallese. ${ }^{3}$ ) Tuttavia l'inglese rimase la lingua ufficiale e di affari nel Paese di Galles, sebbene recentemente la lingua gallese sia ammessa come materia speciale di insegnamento nelle scuole. Perciò non ostante i progressi fatti negli ultimi tempi, specialmente per riguardo alle pubblicazioni nella lingua gallese ${ }^{4}$, la conoscenza dell' inglese si estende ad una gran parte della popolazione e solamente una minoranza non conosce altra lingua che il gallese. Secondo il censimento ufficiale del 1901 gli abitanti del Paese

1) Vedasi per tutto cid Rh y - Brynmor Jones, o. c. p. 50 seg. 2) Cf. D. Evans, The Sunday School in Wales. London 1883; Rees, History of Protestant Nonconformity in Wales. London 1883, 2. ed. p. 394seg.; Sir Thomas Phillips, Wales. London 1849 p. 247-314. - ${ }^{3}$ ) L'Eisteddfod si celebra ora una volta all' anno, promosso dalla National Eisteddfod Association, sotto gli auspici della Honorable Society of the Cymmodorion, con sede principale a Londra. Per la storia dell' Eisteddfod, le cui origini risalgono al sec. XII e per la sua importanza per l'arte e per la letteratura gallese cf. Rhys. Brynmor Jones, o. c. p. 516-524; H. W. Clarke, History of the Church of Wales, p. 174seg. - 4) Dal 1781 al 1800 furono pubblicate nel Paese di Galles 440 opere in gallese e 155 in inglese, dal 1881 al 1895, 1605 opere gallesi e 728 inglesi. Vedansi in proposito le tavole comparative in Rhys-Brynmore Jones, p. 533, e Silvan Evans, Cambrian Bibliography. Lanidloes 1869. 
La separazione dello Stato dalla Chiesa nel Paese di Galles. 257

di Galles di età superiore ai due anni, si distinguono linguisticamente nel modo seguente:

Abitanti che parlano solamente l'inglese 928.222 cioè $49.9 \%$ $" \quad " \quad " \quad$ il gallese $280.905,15.1 \%$ $"$ bilingui . . . . . . . . $648.919,34.8 \%$

Escludendo dal censimento linguistico le contee prevalentemente inglesi del Monmouthshire e Glamorganshire si avrebbero 226. 399 abitanti di lingua esclusivamente gallese, 247.984 di lingua inglese e 322.843 bilingui. $^{1}$ )

Un elemento importante nella cultura nazionale del Paese di Galles fu la riorganizzazione della scuola secondaria mediante il Welsh Intermediate Education Act del 1889, per cui si creava un completo sistema di scuole destinate a preparare gli allievi alle Università e scuole superiori. ${ }^{2}$ ) Verso la metà del sec. XIX risorgeva l'antica questione di un' Università nazionale gallese, e dopo varie vicende veniva fondato nel 1872, per sottoscrizioni private, l'University College of Wales di Aberystwyth. Ad esso seguivano il South Wales College di Cardiff nel 1883 e il North Wales College di Bangor nel 1884, e finalmente con diploma reale del 30 novembre 1893, veniva creata la University of Wales, in cui venivano incorporati $i$ collegi già prima esistenti. ${ }^{3}$ )

In stretta relazione col movimento nazionale del Paese di Galles stanno le sue istituzioni ecclesiastiche, per cui la

1) Togliamo questi dati statistici dal Report of the Royal Commission on the Church of England and other religious Bodies in Wales and Montmouthshire, London 1910 (Cd. 5452, cita.to in seguito Report. 1910) p. 16. 17. Per il precedente censimento del 1891, dove per la prima volta venne rilevato il numero degli abitanti che parlavano solamente gallese e per la critica dei risultati cf. Southall, Wales and her Language, 2. ed. London 1893; I d em, The Welsh Language Census of 1891, Newport 1895. Per i calcoli sui gruppi linguistici nei decennii precedenti vedasi il Report of the Committee on Intermediate Education in Wales, London 1881, p. XLVIJ e Rhys-Brynmore Jones, p. 546 e seg. $-{ }^{2}$ ) Un ampia relazione sull' istruzione secondaria nel Paese di Galles é contenuta nel vol. II dei Special Reports on Educational Subjects, London 1898, pubblicato dall' Educational Department (Cd. 8943). - ${ }^{3}$ ) Cf. Viriamu Jones, The University of Wales, Cardiff 1906, e la publicazione annuale Calendar of the University of Wales, New port. 
tendenza alla separazione fra lo Stato e la Chiesa si ricollega direttamente col risveglio nazionale ed autonomistico degli ultimi decennii.

Il Cristianesimo, importato nel Paese di Galles probabilmente verso il termine dell' occupazione militare romana, si svolge nei primi secoli indipendentemente da influenze esteriori e presenta per questo riguardo una notevole analogia colle chiese celtiche affini della Scozia e dell' Irlanda. ${ }^{1}$ ) Più a lungo peró si mantennero gli elementi celtici nella Chiesa del Paese di Galles $^{2}$ ), che si manifestano specialmente nell' organizzazione monastica e nella tendenza della Chiesa gallese ad affermare la propria autonomia nei varii periodi della sua contrastata e controversa evoluzione storica.

N.el VI secolo si trovano già costituite le sedi episcopali di S. David, Llandaff, Bangor e S. Asaph, mantenutesi fino all' epoca attuale e come $i$ vescovi gallesi cercarono di conservare la loro autonomia di fronte alla missione romana di S. Agostino, così cercarono in seguito di sottrarsi alla supremazia della sede arcivescovile di Canterbury. Ma come la Chiesa del Paese di Galles aveva accettato il sistema romano del calcolo della Pasqua, così la sua organizzazione si venne gradatamente assoggettando a Canterbury, come lo provano le diverse consacrazioni di vescovi delle diocesi gallesi per parte degli arcivescovi di Canterbury, di cui si ha traccia già

1) Cf. H. Soames, The Anglo-Saxon Church, 1835; A. J. Romilly, Monumental History of the early British Church, London 1889; Haverfield, Early British Church, nella English Historical Review, luglio 1896; J. W. Willis Bund, The Celtic Church of Wales, London 1897; Z immer, Keltische Kirche, nella Real-Enzyklopädie für protestantische Theologie und Kirche, di Herzog-Hauck, 3. ed; Hugh Williams, Christian Church in Wales, in Transactions of the Cymmodorion Society, 1893. - 2) Per la storia delle istituzioni ecclesiastiche nel Paese di Galles, oltre le opere citate alla nota precedente ef. Haddan-Stubbs, Councils and ecclesiastical Documents relating to Great Britain and Ireland (4. vol, London 1869-78), Vol. I. p. 299-344; E. G. Newell, History of the Welsh Church, London 1895; H. W. Clarke, A History of the Church of Wales, London 1896; J. F. Lloy d, History of Wales. cit. I. p. 102 . 143. 171.202 ; II. p. 447 . 480.554 . 754 seg. dove si trova una trattazzione veramente scientifica ed imparziale delle varie e contrastate questioni relative alle istituzioni ecclesiastiche gallesi; $W$ arre $n$, Liturgy and Ritual of the Celtic Church, Oxford 1881. 
fin dal IX secolo. Dopo la conquista normanna la fusione della Chiesa gallese con quella anglosassone é agevolata dai diversi vescovi normanni eletti alle sedi gallesi, e nelle lotte per assicurare a queste diocesi titolari di stirpe gallese, si manifestano le tendenze separatistiche della Chiesa del Paese di Galles, di cui i principi gallesi miravano a servirsi come di elemento atto a rafforzare l'autonomia nazionale. L'episodio di Geraldo Cambrense (Gerald de Barri o Gerald the Welshman), celebre nella storia ecclesiastica del Paese di Galles ${ }^{4}$ ) e la nota petizione dei principi gallesi ad Innocenzo III ${ }^{5}$ ), diretta contro l'autorità arcivescovile di Canterbury, sono espressione di queste tendenze. In seguito la Chiesa del Paese di Galles viene gradatemente attratta nell' orbita dell' organizzazione ecclesiastica inglese, sebbene la fusione non sia sempre stata completa ed uniforme, per cui riti ed elementi celtici e gallesi si conservarono nelle istituzioni religiose del Paese di Galles. All' epoca di Enrico VIII la Riforma anglicana vi fu estesa senza difficoltà e la dissoluzione dei monasteri e delle abbazie fu compiuta senza ostacoli. Una legge di Elisabetta (5. Eliz. c. 28) ordinava la traduzione in Gallese della Bibbia $\mathrm{e}$ in tal modo la Riforma contribuiva indirettamente a mantenere e diffondere efficacemente la lingua nazionale gallese. ${ }^{6}$ ) Inoltre la

1) Per le peculiari caratteristiche dell' organizzazione dei monasteri nel Paese di Galles, cf. Willis Bund, The Celtic Church, cit. cap. IV. p. 145-206; l'importanza di questo elemento monastico non é forse sufficientemente rilevata nel notevole articolo di A. N. Palmer, The portionary Churches of Medieval North Wales, in Archeologia Cambrensis, 1886, p. 175-209. - 2) Cf. W. Bevan, St. David, 1888; Dr. T hom as, St. Asaph. 1888. W. B. Jones and E. A. Freemann, The History and antiquities of St. David's 1856. Vedasi anche Clarke, A Hist. of the Church in Wales, cit. p. 7 seg. $-{ }^{3}$ ) Vedasi per la questione della Pasqua H. W. Clarke, o. c. p. 22 e seg.; Giry, Manuel de diplomatique, Paris 1894, p. 141-54. - ") Cf. J. S. Brew er, Giraldus Cambrensis, 7. vol. London 1861-77 (nella Public Rolls Series); Dictionary of National Biography, 2. ed. s. v. Giraldus Cambrensis, colla letteratura ivi citata. - ${ }^{\text {s) }}$ In Brewer, Gir. Cambr. Opera, cit. III. p. 244. - ${ }^{\circ}$ I dieci comandamenti e il credo furono tradotti in gallese nel 1546 da Sir John Price; nel 1567 veniva pubblicata la traduzione del Nuovo Testamento di William Salesbury, a cui seguiva nel 1588 la versione dell' Antico Testamento del Dr. William Morgan. Vedasi 
Regina Elisabetta nominò alle sedi vescovili gallesi numerosi vescovi di origine e di lingua gallese, e questa pratica continuò fino al principio del sec. XVIII, quando col regno della Regina Anna fu iniziata la pratica della nomina di vescovi inglesi alle sedi gallesi. $\left.{ }^{1}\right) \quad \mathrm{Ma}$ il fatto che le diocesi gallesi servivano solo come sedi di passaggio ai vescovati inglesi indeboliva grandemente l'opera dell' episcopato del Paese di Galles, ed a ciò si aggiungevano gli abusi del cumulo di benefici e l'assenteismo dei prelati ${ }^{2}$ ), che prepararono il terreno a quel moto nonconformista del sec. XVII, il quale ebbe una profonda importanza religiosa e nazionale per il Paese di Galles. Delle varie fasi della storia ecclesiastica gallese, questa che segna il sorgere e il diffondersi delle diverse confessioni dissidenti, é la più direttamente collegata alla questione della separazione dello Stato dalla Chiesa nel Paese di Galles.

Nel 1638 William Wroth, rettore di Llanvaches, veniva privato dal suo beneficio, per questioni dogmatiche, $e$ in seguito costituiva la Chiesa Congregazionalista di Llavanches, divenendo cosi il capo e fondatore degli Indipendenti o Congregazionalisti del Paese di Galles. A lui si univano William Erbury $\Theta$ Walter Craddock, che erano parimenti stati espulsi dalla Chiesa anglicana ed una serie di riformatori del sec. XVII, fra cui Vavasor Powell, Morgan Lloyd, Hugh Owen e James Owen continuarono l'opera loro a cui fu dato un efficace impulso dall' Act of Toleration del 1689. Durante il sec. XVII il moto nonconformista non aveva avuto un

il Dictionary of National Biography alle relative biografie e Rees, History of Protestant Nonconformity in Wales, cit. p. 13. seg.; Dr. Thomas Llewelyn, An historical Account of the British or Welsh Versions and Editions of the Bible, 1768.

1) Vedasi per ciò H. W. Clarke op. cit. p. 73-78 e p. IX-XXIV, dove é l'elenco completo dei vescovi dal Paese di Galles dall' inizio del regno di Elisabetta, coll' indicazione della loro nazionalità. Dal 1715 al 1859 non vennero nominati titolari gallesi, mentre dal 1870 in poi, per opera del Gladstone e del Disraeli, veniva ripresa la tradizione dei vescovi nazionali gallesi. - ${ }^{2}$ ) Ampiamente in proposito $\mathrm{H}$. W. Clarke o. c. p. 79-94; cf. anche H. T. Edwards, Wales and the Welsh Church, London 1869, p. 321 seg. e A. J. Jo hnes, The causes of Dissent in Wales, London 1831, nuova ediz. 1870. 
La separazione dello Stato dalla Chiesa nel Paese di Galles. 261

vero e proprio carattere popolare e nazionale, ma si era diffuso essenzialmente fra le classi più alte della popolazione, che erano prevalentemente inglesi 0 anglicizzate. ${ }^{1}$ ) Inoltre il letargo della vita ecclesiastica e spirituale nel Paese di Galles, in questo periodo, si rispecchiava non solo nelle condizioni della Chiesa anglicana, a cui abbiamo sopra accennato, ma negli scarsi effetti della predicazione nonconformista rappresentata specialmente dai Battisti e dagli Indipendenti. ${ }^{2}$ ) All' opera di Griffith Jones (1684-1761), il fondatore delle Circulating Schools, di Howell Harris (1714-1773), e di Daniel Rowlands (1713-1790) é dovuto il grande risveglio della confessione metodista, che doveva avere una profonda influenza sullo sviluppo e sui progressi del Nonconformismo nel Paese di Galles. ${ }^{3}$ ) Esso andò congiunto con un notevole risveglio nazionale ed intellettuale, a cui é dovuta essenzialmente la conservazione e diffusione della lingua gallese, l'aumento delle opere pubblicate in gallese e lo stimolo per una più intensa cultura nazionale.4) Ed indirettamente, per il naturale spirito di concorrenza e di emulazione, il moto nonconformista, contribuiva a rendere più efficace e intensa l'opera della Chiesa anglicana, che adottava in parte la lingua gallese, cercando di attrarre nella sua orbita gli elementi nazionali gallesi. ${ }^{5}$ )

Ma col rafforzarsi delle confessioni nonconformiste e col$l$ 'intensificarsi della loro opera in linea non solo religiosa ma nazionale, veniva affermandosi la tendenza a togliere alla Chiesa anglicana $i$ suoi privilegi e la sua posizione di Chiesa di Stato. Il postulato della separazione fra la Chiesa e lo Stato nel Paese di Galles veniva sempre più insistentemente

1) Questa circostanza che non venne sempre adeguatamente considerata trovasi giustamente lumeggiata in Rhys-Brynmor Jones, o. c., p. 462 . - 2) Cf. Lecky, History of England in the XVIII Century, London 1888, Vol. II p. 602-604. - 3) Per le speciali caratteristiche dogmatiche della confessione metodista del Paese di Galles, detta anche metodista-calvinista, di fronte al metodismo di Wesley cf. H. W. Clarke, o. c. p. 158-165, e T. Darlington, The Church and Nonconformity in Wales, nella Contemporary Review, Maggio 1894. 4) Cf. Rhys-Brynmor Jones, o. c. p. 475-477. - s) Per l'opera della Chiesa anglicana in questo senso ef. H. T. Edwards, Wales and the Welsh Church, cit. 
sostenuto dai Nonconformisti del Paese di Galles, che affermarono essere la Chiesa Anglicana un' istituzione estranea e contraria allo spirito nazionale gallese ed alle sue aspirazioni. ${ }^{1}$ )

La questione della separazione della Chiesa anglicana dallo Stato veniva portata al Parlamento inglese fino dal 1870, quando al 24 maggio di quell'anno Mr. Watkin Williams (in seguito Judge Williams), presentava una risoluzione che proponeva la separazione per il Paese di Galles e l'impiego dell' avanzo della dotazione della Chiesa anglicana, dopo che fosse stato provvisto ai diritti acquisiti, per un sistema di istruzione non confessionale nel Paese di Galles. ${ }^{2}$ ) Consimili risoluzioni venivano proposte a diverse riprese ${ }^{3}$ ), finchè nel 1893 veniva presentato alla Camera dei Comuni il Suspensory Bill, a cui seguiva nel 1895 il Welsh Disestablishment and Disendowment Bill presentato dal Ministro degli interni (Home Secretary) Mr. Asquith. Ma lo scioglimento del Parlamento poneva termine al progetto prima che venisse in discussione.

A base dell' azione parlamentare suaccenuata, l'elemento nonconformista poneva il principio che la dotazione della Chiesa anglicana risaliva ad un' epoca in cui essa rappresentava l'intera nazione, ma che ciò non aveva più ragione di esistere all' epoca attuale, quando essa non era che una frazione delle varie confessioni del Paese di Galles. D'altra parte la Chiesa anglicana faceva valere i progressi dell' opera sua nelle diocesi gallesi, affermando, in base alla continuità storica, l'unione organica, all' epoca presente, fra la Chiesa anglicana in generale e le istituzioni di essa nel Paese di

1) In questo senso é dettato il libro di H. W. Clarke, A History of the Church of Wales cit. specialmente cap. 30 e 32, p. 167. $178 \mathrm{seg}$. I gravami gallesi contro la Chiesa anglicana si trovano anche vigorosamente espressi in Sir G. O. Morgan, The Church of England and the People of Wales, 2. ed. 1895. - 2) Cf. H. W. Clarke, History of the Church of Wales, p. 221 e seg. dove é l'elenco di tutte le risoluzioni presentate in proposito al Parlamento inglese dal 1870 in poi. - ${ }^{3}$ ) Cosi al 9 marzo 1886 (Mr. Dillwyn), 14. maggio 1889 (idem), 20 febbr. 1891 (Mr. Pritchard Morgan), 1892 (Mr. Samuel Smith). 
La separazione dello Stato dalla Chiesa nel Paese di Galles. 263

Galles. E mentre per una parte si formava il Central Church Committee for Defence and Instruction, per salvaguardare la posizione ufficiale della Chiesa di Stato anglicana, i Nonconformisti continuarono attivamente l'opera di propaganda per la separazione, servendosi come organo centrale del National Council of Evangelical Churches. $\left.{ }^{1}\right) \mathrm{Da}$ entrambe le parti la polemica venne condotta con molta vigoria e accompagnata da una larga agitazione di propaganda improntata spesso da un' acre intransigenza, a cui non furono sempre estranei gli aspetti politici della questione, per il fatto che il partito liberale inglese aveva accolti i postulati dei Nonconformisti gallesi per la separazione della Chiesa dallo Stato, che invece sono avversati dalla grande maggioranza del partito conservatore. Ma uno dei punti più difficili della controversia é lo stabilire con esattezza, anche approssimativa, il rapporto numerico delle diverse confessioni nel Paese di Galles. Come é noto nelle schede del censimento inglese non é compresa l'indicazione della confessione, ed a ciò si opposero costantemente i Nonconformisti, sostenendo che un censimento religioso fosse contrario ai principii fondamentali del Nonconformismo, come quello che costituiva un' ingerenza dello Stato nella libertà di coscienza individuale, ed affermando inoltre il poco valore statistico, che in pratica esso arrebbe avuto. ${ }^{2}$ )

Per sopperire a questa mancanza di dati statistici per riguardo alle diverse confessioni nel Paese di Galles e fornire una guida sicura all' azione parlamentare relativa alle istituzioni ecclesiastiche gallesi veniva istituita nel 1906 una «Commissione reale» che doveva riferire sulle origini, natura,

1) Contro la separazione vedasi, oltre diverse pubblicazioni di carattere occasionale, foglietti e opuscoli di propaganda, Dr. Edwards, (Vescovo di St. Asaph), A Handbook on Welsh Church Defence. London 1895. 3. ediz. In senso separatista H. W. Clarke, The Case for Disestablishment, London 1894; J. Morgan Gibbon, Weighed in the Balance The Case for Welsh Disestablishment. s. a [1910]. Vedasi anche Sir Thomas Phillips, Wales, London 1849 e A. J. Johnes, The Causes of Dissent in Wales, London 1831, nuova ed. 1870. cit.; H. Elvet Lewis, Nonconformity in Wales (nella serie Eras of Nonconformity edita da G. Silvester Horne. - ${ }^{2}$ ) Vedasi al riguardo J. Morgan Gibbon, Weighed in the Balance, etc. cit., p. 62. 
importo ed uso delle temporalità, dotazioni ed altre proprietã della Chiesa anglicana nel Paese di Galles e Monmouthshire e sull' opera spiegata dalle Chiese di qualsiasi denominazione in detti territorii. ${ }^{1}$ ) La Commissione Reale dopo 134 sedute, in cui furono esaminati 135 testimonii delle diverse confessioni pubblicava una monumentale relazione ${ }^{2}$ ), che resterà memorabile nella storia del Paese di Galles, come la classica relazione della «Royal Commission on Land in Wales and Monmouthshire», sebbene, come si vedrà, i suoi risultati siano stati assai controversi. L'esame della Commissione Reale fu rivolto alla Chiesa anglicana, alle quattro grandi confessioni nonconformiste cioè Battisti, Metodisti Calvinisti (detti anche Chiesa presbiteriana del Paese di Galles), Congregazionalisti o Indipendenti e Metodisti Wesleyani, ed alle confessioni meno numerose del Paese di Galles (cioè Cattolici, Fratelli di Plymouth, Chiese di Cristo, Chiesa presbiteriana d'Inghilterra, Metodisti primitivi, Società di amici, Unitarii, Chiesa metodista Unitaria e infine l'Esercito della Salute).

I risultati principali delle indagini possono riassumersi nei dati che esponiamo qui sommariamente.

Per riguardo alla proprietà ecclesiastica della Chiesa anglicana nel Paese di Galles, la Commissione constatava

1) La Commissione reale detta comunemente "Royal Commission on the Church of England and other religious Bodies in Wales and Monmouthshire" veniva istituita con Decreto Reale del 21 giugno 1906, che chiamava a farne parte Sir Roland Iomaie Bowdler Waughan Williams (Presidente), Hugh Richard Heathcote Cecil, Sir John Williams, Frank Edwards, Owen Evans, Samuel Thomas Evans, Andrew Martin Fairbairn, John Ernest Greaves ed Henry Jones. In seguito alle dimissioni di S. T. Evans, A. M. Fajrbairn ed H. Jones con altro Decreto Reale del 1 maggio 1907 venivano loro sostituiti Sir David Brynmor Jones, John Herbert Davies e John Morgan Gibbon, per cui nella commissione venivano a trovarsi cinque fautori dichiorati della separazione nele Paese di Galles e cioè Sir John Williams, Sir Francis Edwards, Sir D. Brynmor Jones, Rev. S. Morgan Gibbon e J. H. Davies. - 2) La voluminosa relazione pubblicata nella forma di diversi libri azzurri (blue books) si compone dalle seguente parti: Report of the Commission (Gd. 5432); Minutes of Evidence 3. vol. (Cd. 5433-35); Subiect and Witness Indexes to Minutes of Evidence (Cd. 5439); Statistic and other Appendices to the Evidence. 3. vol. (Gd. 5436-38), London (Wymann and Sons) 1910. 
La separazione dello Stato dalla Chiesa nel Paese di Galles. 265

l'impossibilità di procedere ad un esame storico legale dell'origine delle singole dotazioni ${ }^{1}$ ), ma stabiliva $\mathrm{i}$ cespiti del patrimonio delle 1.014 parrocchie del Paese di Galles²) distinguendo quelli in cui il titolare del beneficio non ha alcun interesse pecuniario (fabbriche ecclesiastiche e cimiteri, dagli altri in cui il beneficiato è direttamente interessato e cioè; case parrocchiali, fondi beneficiorii (Glebes), decime (Tithe Rent Charge), intraiti diversi, comprese le sovvenzioni del Queen Anne's Bounty e degli Ecclesiastical Commissioners, dotazioni a scopi specifici, sovvenzioni degli Ecclesiastical Commissioners per provvedere al clero assistente. ${ }^{3}$ ) La dotazione dei benefici veniva calcolata dalla Commissione in $£$ st. 242. 669, di cui $£$ st. 135.980 ritenute esistenti prima del $1703, £$ st. 37.344 date dal Queen's Anne Bounty e $£$ st. 49.669 date dagli Ecclesiastical Commissioners. Il patrimonio diocesano, il cui reddito é di $£$ st. 35. 609 è amministrato dagli Ecclesiastical Commissioners. ${ }^{5}$ )

1) Report 1910 p. 7 seg. - 2) La grande maggioranza dalle parrocchie del Paese di Galles appartiene alle quattro diocesi gallesi di Bangor, Llandaff, S. Asaph e S. David. Solo alcune appartengono a diocesi inglesi e cioè Chester (3), Hereford (20) e Lichfield (1) cf. Report cit. p. 6. - ${ }^{3}$ ) Per questi diversi elementi componenti il patrimonio ecclesiastico della Chiesa anglicana ef. Phillimore, The Ecclesiastical Law of the Church of England, London 1895. 2. ed. e H. Hardy, The Benefices Act etc., London 1899. - 4) Nel 1703 venivano costituiti in corporazione $i$ "Governors of the Bounty of Queen Anne for the augmentation and maintenance of the poor Clergy" chiamati a distribuire per il miglioramento degli stipendii del clero $i$ redditi delle annate e decime che Eurico VIII., abolita l'autorità pontificia, areva devolute alla corona (26. Henry VIII. c. 3). Gli Ecclesiastical Commissioners, istituiti nel 1835 per vigilare sul patrimonio ecclesiastico colle leggi 5.6 William IV. c. $30 ; 6$. 7 William IV. c. 7 divennero poi gli amministratori del fondo costituito in seguito alla riorganizzazione delle diocesi e capitali cattedrali, specialmente per le leggi 3. 4 Vict. c. 113 e 23. 24 Vict. 124. Vedasi per tutto ciò P hillimore, cit. e Cripps, A practical Treatise on the Law relating to the Church and Clergy, London 1863. 4. ed. p. 46 seg. - - ') Vedansi anche i dati contenuti nel "Return of the gross Incomes, derived from permanent Endowments of Benefices situated wholly or partly in Wales and Monmonthshire etc." di Sir Lew is Dibdin nel Report, 1910, Vol. I. Parte II. app. A. 
Per riguardo al rapporto numerico delle varie confessioni, in base agli annuarii ufficiali delle confessioni stesse, alle statistiche da esse presentate ed alle diverse testimonianze assunte, la Commissione ${ }^{1}$ ) giunse ai risultati espressi dalla seguente tavola:

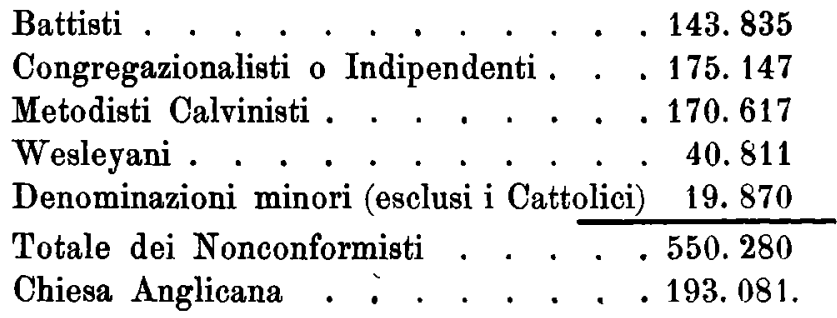

Per riguardo ai Cattolici secondo un memoriale presentato alla Commissione dal Vescovo di Menevia, il numero degli appartenenti alle Chiese cattoliche del Paese di Galles é di 64.800, con 142 sacerdoti residenti. ${ }^{2}$ )

Per riguardo al clero, di cui vennero studiate minutamente, la classificazione, la preparazione e le funzioni, la Commissione fossava le seguenti cifre:

$\begin{array}{lll}\text { Chiesa anglicana . } & \text {. } & 1597 \\ \text { Metodisti calvinisti } & . & 1131 \\ \text { Wesleyani . . . } & \text {. } & 1158 \\ \text { Battisti . . . . } & \text {. } 1171 \\ \text { Congregazionalisti } & \text {. } & 1344\end{array}$

Per riguardo agli stipendii del clero, essi vengono calcolati in media circa $£$ st. 250 per i beneficiati della Chiesa anglicana, mentre per le chiese nonconformiste sono

1) Report, cit. p. 20. La principale difficoltà di questa statistica consiste nel determinare i requisiti di appartenanza alle singole confessioni. Per la Chiesa anglicana fu adottato il principio di computare $\mathrm{i}$ «comunicants», ciò̀ quelli che si accostano alla comunione almeno una volta all' anno (cf. Report, p. 19. 25). Per le confessioni nonconformiste le difficoltà statistiche sono anche maggiori per la distinzione, non sempre precisa e sicura, fra «members» e «adherents» (Report, p. 25). - ${ }^{2}$ ) Report, p. 42. Nel 1885 Papa Leone XIII costituiva il Vicariato del Paese di Galles, trasformato poi nella Diocesi di Meneria al 12 maggio 1898. - ${ }^{3}$ ) Report, p. 43-51. 
assai variabili, per la circostanza che $\mathrm{i}$ ministri non sono fissi, ma ufficiano in diversi luoghi, ricevendo un onorario dalle congregazioni locali. Solo i Wesleyani pagano i ministri da un fondo centrale, con una somma variabile da $£$ st. 130 a 200 .

Una delle parti più caratteristiche della relazione della Commissione ${ }^{1}$ ) é quella relativa agli edifici di culto, per riguardo ai posti (A c comodation), questione assai dibattuta in quanto le statistiche relative ai posti nelle chiese e cappelle, furono spesso oggetto di vivaci controversie. Secondo la Commissione il numero dei posti é il seguente:

\begin{tabular}{|c|c|c|}
\hline & \\
\hline & & \\
\hline & & 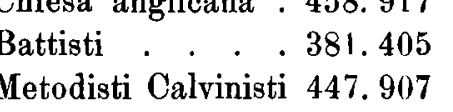 \\
\hline & & \\
\hline & \\
\hline \multirow{2}{*}{\multicolumn{3}{|c|}{$\begin{array}{l}\text { Cattolic } \\
\text { Diversi }\end{array}$}} \\
\hline & & \\
\hline \multicolumn{3}{|l|}{ tale } \\
\hline
\end{tabular}

Le conclusioni della Commissione al riguardo, sono che il numero dei posti é eccessivo, di fronte al numero degli abitanti, per il fatto che per 2.012.617 abitanti si hanno 2.019.151 posti negli edifici di culto, mentre si deve tener calcolo dei bambini che non vengono condotti ai servizi divini, e delle diverse persone, che per varie cause non li frequentano. Specialmente venne rilevato che l'eccesso del numero dei posti si riscontra nelle Chiese nonconformiste e ciò fu attribuito dalla Commissione agli sforzi fatti dalle singole comunità per estendere la propria sfera dazione, spesso in una misura non proporzionata alle condizioni di fatto. Una parte specialmente notevole della relazione ${ }^{2}$ ) si riferisce alle scuole domenicali (Sunday schools). Esse costituiscono una delle istituzioni più caratteristiche delle diverse confessioni del Paese di Galles, e furono uno dei mezzi più efficaci della propaganda nonconformista, in quanto vengono largamente frequentate

1) Report, p. 34--58. L'eccesso dei posti in proporzione alla frequentazione dei luoghi di culto, viene designato tecnicamente col termine di „overlapping“ (ib. p. 58). $-{ }^{2}$ ) Report, p. 58-68. 
non solo da fanciulli, ma anche da adulti. Cosi le scuole domenicali nonconformiste furono frequentate $\mathrm{da} 328.878$ fanciulli (inferiori ai 15 anni) e 281.857 adulti, di fronte a 56. 088 fanciulli e 112.698 adulti che frequentano le scuole domenicali della Chiesa anglicana.

La commissione ${ }^{1}$ ) ha rilevata poi la importanza di un' azione intesa ad estendere ed intensificare l'opera ecclesiastica delle diverse confessioni, che fu detta Forward Movement e Church extension, e che si esplicò non solamente in una più vasta propaganda religiosa, ma anche in un' efficace opera sociale. Per quanto riguarda la cooperazione fra le diverse confessioni, la Commissione ha constatata un' ampia comunità di opere, specialmente riguardanti il servizio divino, fra le confessioni nonconformiste, che si è venuta accentuando negli ultimi tempi. Per contro la Chiesa anglicana non ha relazioni coi Nonconformisti per quanto si riferisce al servizio divino, mentre coopera con essi, in materie morali e sociali, riguardanti l'intera popolazione. Per riguardo alla posizione delle diverse istituzioni ecclesiastiche gallesi di fronte alle grandi confessioni dell'Inghilterra, fu osservato che le quattro diocesi gallesi della Chiesa anglicana (Bangor, Llandaff, S. Asaph e S. David) costituiscono altrettante unità della provincia ecclesiastica di Canterbury e non differiscono nei caratteri generali, dalle diocesi inglesi. A loro volta le confessioni nonconformiste gallesi (eccetto i cosidetti Strict Baptist) appartengono al National Council of Evangelical Free Churches of England and Wales, che contava nel 1908 quattro Federazioni distrettuali e 167 Concilii locali gallesi. L'elemento locale, meno che per i Metodisti Calvinisti, non é rappresentato nelle congregazioni nonconformiste gallesi, da speciali caratteristiche, per cui l'elemento che essenzialmente distingue le confessioni nonconformiste gallesi da quelle dell' Inghilterra é sopratutto l'elemento linguistico. A questo riguardo si deve tener presente la prevalenza della lingua gallese nei servizi divini nonconformisti nel Paese di Galles, ma tuttavia anche le

1) Report, p. 68-74. - 2) Report, p. 26 seg. per la Chiesa anglicana; p. 74-76 per le confessioni nonconformiste. 
La separazione dello Stato dalla Chiesa nel Paese di Galles. 269

confessioni nonconformiste vi provvedono servizii divini inglesi - bilingui. Invece la lingua inglese prevale nei servizii divini della Chiesa anglicana nel Paese di Galles, che provvede 2.393 servizii domenicali inglesi, di fronte a 1103 gallesi e 228 bilingui.

Le difficoltà che erano sorte nel corso dej lavori della Commissione reale e che avevano portato alle dimissioni di diversi dei suoi membri, si manifestarono anche nella redazione della relazione, che non venne firmata da tutti i commissarii, e da altri solo con esplicite riserve e con l'annuncio di memoriali speciali. Cosi alla relazione principale si trovano aggiunti memoriali dell'Arcidiacono Owen Evans e Lord Hugh Cecil $^{1}$ ), di Mr. John H. Davies ${ }^{2}$ ), del Rev. J. Morgan Gibbon ${ }^{3}$ ), e di Sir John Williams. ${ }^{4}$ ) In essi i dati della relazione, specialmente riguardo alle statistiche delle diverse confessioni, sono variamente criticati dal punto di vista delle singole confessioni in questione. $\mathrm{Ma}$ se con ciò fu in parte diminuito il valore generale della relazione, essa rimarrà tuttavia un documento di grande importanza per la storia delle istituzioni ecclesiastiche gallesi, mentre i memoriali speciali dei comissarii resteranno come la testimonianza dell' attitudine dei varii gruppi religiosi, di fronte alle grandi controversie ecclesiastiche del Paese di Galles.

La questione della separazione fra lo Stato e la Chiesa nel Paese di Galles ha essenzialmente una base finanziaria, poichè sopratutto non si tratta solamente di togliere alla Chiesa anglicana la sua posizione di Chiesa di Stato (Disestablishment), ma di devolvere una gran parte della sua dotazione ad altri scopi (Disendowment). ${ }^{5}$ )

Per una parte la Chiesa anglicana fa valere la sua prevalenza numerica di fronte alle singole confessioni non-

1) Il Memorandum dell' Arcidiacono Evans e Lord Hugh Cecil (Report, p. 80-158) rappresenta l'attitudine della Chiesa anglicana ed é specialmente rivolto contro le statistiche dei Nonconformisti. 2) Nel Report, p. 159-176. - 8) Report, p. 177-185. - 4) Oltre il Report di Sir John Williams (l. c. p. 247-395) fu aggiunto alla relazione (ibid. p. 187-237) un memorandum preparato da Sir John Williams e firmato da Sir Francis Edwards, Sir David Brynmor Jones e dal Rev. J. Morgan Gibbon. - ${ }^{5}$ ) Cf. A. E. Freeman, Disestablishment and Disendowment, London 1891. 
conformiste, l'opera spiegata in linea ecclesiastica e sociale, non solo ma anche in linea nazionale, specialmente dopo che negli ultimi tempi i vescovi nel Paese di Galles furono sempre di nazionalità gallese, e il fatto che la Chiesa anglicana delle provincie gallesi forma un tutto organico colla Chiesa anglicana generale. ${ }^{1}$ ) Dal canto loro i Nonconformisti affervano la maggioranza numerica delle loro confessioni, prese collettivamente, di fronte alla Chiesa anglicana, il carattere straniero di questa, di fronte alla nazionalità gallese e la circostanza che la sua dotazione, concessale quando era l'unica chiesa esistente nel Paese di Galles, non ha più ragione di essere ora che sono sorte e si sono vigorosamente affermate altre confessioni, rappresentanti più direttamente le aspirazioni nazionali e religiose della gran parte della popolazione indigena gallese. ${ }^{2}$ )

Ma anche per il suo substrato politico, a cui abbiamo sopra accennato, la questione, oltre ad essere semplicemente determinata da elementi ecclesiastici, ha una base profondamente nazionale, e come tale si ricollega alle tendenze separatistiche ed autonomiste del Paese di Galles, che si vennero sempre più chiaramente delineando negli ultimi tempi, per il risveglio delle correnti intellettuali e letterarie gallesi.

Il progetto di separazione presentato alla Camera dei Comuni al 21 aprile 1909 si intitola «A Bill to terminate the Establishment of the Church of England in Wales and Monmouthshire, and to make provision in respect of the Temporalities thereof, and for other purposes in connection with the matters aforesaid» o più brevemente "Welsh Church Bill». Esso consta di 34 articoli ed é calcato nelle linee generali sul progetto del 1895 e sulla legge di separazione fra lo Stato e la Chiesa anglicana in Irlanda del 1869.

L'articolo 1. dispone che alla data fissata per la separazione la Chiesa anglicana, in quanto si estende ed esiste nel Paese di Galles e Monmouthshire, deve cessare di essere

1) La posizione della Chiesa anglicana é affermata nel citato memoriale dell' Arcidiacono Evans e di Lord Hugh Cecil. - 2) Per l'attitudine delle confessioni nonconformiste cf. J. Morgan Gibbon, Weighed in the Balance, cit. 
stabilità per legge e che dopo l'approvazione della legge, nessuno dovrà essere nominato dal Re o da qualsiasi persona ad uffici ecclesiatici gallesi, in base a qualsiasi diritto di patronato esistente. Contemporaneamente, alla data della separazione, vengono sciolte tutte le corporazioni ecclesiastiche e cattedrali, tanto singole che aggregate ${ }^{2}$ ), della Chiesa anglicana nelle diocesi gallesi; $i$ vescovi di queste sedi cessano di far parte della Camera dei Lordi ${ }^{3}$ ), ma conservano a vita, alla stessa guisa degli altri attuali dignitarii della Chiesa anglicana nel Paese di Galles, il loro titolo e diritti di precedenza (Art. 2). Per tal modo cessa il tradizionale legame fra lo Stato e la Chiesa anglicana nelle contee gallesi e la costituzione di questa e la sua posizione legale vengono regolati da un' apposita sezione del progetto. Dal momento della separazione il diritto ecclesiastico cessa di esistere come legge, ma lo stesso diritto, cogli annessi articoli, dottrine e norme disciplinari della Chiesa anglicana, compresa la giurisdizione dell' Arcivescovo di Canterbury esercitata dal tribunale archiepiscopale ${ }^{4}$ ), restano in vigore come norme puramente contrattuali e possono farsi valere davanti ai tribunali ordinarii, in quanto si riferiscono alla proprietà ecclesiastica, peraltro nessun tribunale o autorità ecclesiastica della Chiesa anglicana nel Paese di Galles avrà giurisdizione coercitiva, nè

1) Bill n. 157. Cf. in proposito R. W. Fowel and L. George Dibd in, The Welsh Disestablishment Bill 1909 with explanatory Notes etc., London 1909 (Church Comittee for Defence and Instruction). 2) «Every cathedral and ecclesiastical corporation in the Church in Wales, whether sole or aggregate» Sotto la denominazione «corporation sole» il diritto ecclesiastico inglese conprende il vescovo, decano, arcidiacono, parroco, e i titolari di certe dignità, come canonicati o prebende, aventi un ufficio con successione perpetua, tenuto da una persona sola. Le disposizioni degli art. 1-2 corrispondono a quelli della legge di separazione irlandese del 1869. Però fu osservato che il caso del l'Irlanda era diverso, in quanto tale legge non faceva che sciogliere l'incorporazione della Chiesa anglicana d'Irlanda a quelle dell' Inghilterra avvenuta nel 1800 per mezzo dell' Union Act (39-40 Geo. IIJ. c. 67 art. 5). - - ${ }^{3}$ ) 11 numero dei Lords Spiritual (arcivescovi e vescovi) appartenenti alla Camera alta, viene dalle disposizioni del progetto (art. 2 a. 3) ridotto a ventidue. - ") Questa disposizione corrisponde alla sez. 20 della legge di separazione irlandese, che però non fa cenno della giurisdizione dell' Arcivescovo di Canterbury. 
sarà ammesso l'appello al Re in Consiglio da tali autorità. Nel caso che la Chiesa anglicana nel Paese di Galles intenda, dopo la separazione, adottare nuove norme e costituzioni, esse non potranno farsi valere di fronte all' Arcivescovo, ove questi non vi abbia dato il proprio consenso, che potrà venir dato coll' approvazione del Re in Consiglio. Tali modificazioni agli statuti esistenti, tranne quelle dei formularii rese necessarie dalla separazione, non saranno obbligatorie per quelle persone ecclesiastiche, aventi per la legge di separazione diritti acquisiti, quando entro un mese dall' approvazione di dette nuove norme, abbiano elevata formale protesta per iscritto, davanti alle autorità competenti (Art. 12). Per riguardo alla nuova costituzione della Chiesa anglicana nel Paese di Galles l'art. 13 dispone che nessuna legge o consuetudine debba impedire ai vescovi, al clero e ai laici della Chiesa anglicana di tener sinodi, eleggere rappresentanti e di stabilire costituzioni e norme pel Governo della Chiesa anglicana gallese e per la sua rappresentanza. É questa una disposizione importantissima, in quanto per una parte toglie le restrizioni relative alla convocazione di sinodi contenute nella legge 25 Henry VIII. c.19 e mira alla costituzioni degli enti diocesani e parrocchiali (representative Bodies), a cui vanno devoluti gli edifici di culto ed annessi dell' attuale Chiesa anglicana.

Per attuare la separazione, in quanto riguarda specialmente il patrimonio ecclesiastico, vengono istituiti i Commissarii Gallesi (Welsh Commissioners) costituenti ${ }^{1}$ ) la corporazione dei «Commissioners of Church Temporalities in Wales» (art. 8-10). La durata di tale corporazione é

1) I Commissarii vengono dapprima nominati nella legge, e in caso di successiva vacanza, vengono nominati con decreto reale. Il presidente ha uno stipendio annuo di $£$ st. 1500 , uno dei commissarii $\mathfrak{E}$ st. 1000 , mentre la carica di un altro commissario é gratuita. I commissarii retribuiti e gli altri impiegati della Commissione non possono far parte del Parlamento e sono ineleggibili per la durata delI' ufficio (art. 8). Le facoltà giurisdizionali dei Commissarii, che possono decidere tutte le questioni di fatto o di diritto derivanti dalla legge, ed hanno per le loro inchieste le facoltà spettanti ad un' alta corte sono regolate dall' art. 9. Le questioni relative agli elementi delle «elargizioni private» sono soggette ad appello al Re in Consiglio (art. 10). 
fissata a cinque anni, dopo di che le loro funzioni passano al «Consiglio del Paese di Galles» (Council of Wales) da istituirsi con decreto reale e che dovrà comporsi di rappresentanti delle contee e delle città (art. 11).

Al momento della separazione verranno devolute ai Commissarii Gallesi, tutte le proprietà degli Ecclesiastical Commissioners e del Queen Anne's Bounty, di origine gallese ${ }^{1}$ ) e tutte le proprietà appartenenti a qualsiasi ufficio ecclesiastico o corporazione cattedrale della Chiesa anglicana nel Paese di Galles, soggetti però agli oneri, pesi ed obblighi esistenti, ed alle norme per compensi stabilite nella legge (art. 3). Però la suppellettile mobile delle diverse chiese, che è destinata alla celebrazione degli uffici divini, non si devolve ai Commissari, ma passa alle «nuove rappresentanze» suaccennate, di cui all' art. 13, ed ove queste non siano ancora costituite al momento della separazione la detta suppellettile di culto, resta adibita, senza alcun mutamento o devoluzione, agli stessi scopi cultuali (art. 3 n. 2).

Le norme relative alla parte dell' attuale dotazione che é destinata a rimanere alla Chiesa anglicana dopo la separazione sono contenute nell' art. 6. Esso dispone che $\mathrm{i}$ Commissarii gallesi debbono su domanda delle relative «rappresentanze» trasferir loro 1 . tutte le chiese. 2. le residenze ecclesiastiche colla relativa suppellettile. 3 . i fondi o dotazioni, destinati speeialmente al mantenimento delle chiese o residenze ecclesiastiche. 4. Tutti i cimiteri chiusi, prima della legge di separazione, in conseguenza di legge o decreto reale. 5. le largizioni private (Private Benefactions). Come tali, secondo l'art. 5, devono considerarsi tutti $i$ beni o redditi donati da privati o raccolti a mezzo di pubbliche sottoscrizioni dall' anno 1662 in poi, comprendendosi in esse $i$ beni o redditi di cui non possa determinarsi l'origine, come pure quei fondi appartenenti agli Ecclesiastical Commissioners od al Queen Anne's Bounty, che rientrano in questa categoria. I cimiteri e i beni beneficiarii (Gle bes), vengono assegnati dall'art. 6 agli attuali titolari dei singoli bene-

1) Diverse disposizioni relative ai rapporti amministrativi degli Ecclesiastical Commissioners e del Queen Anne's Bounty sono contenute negli articoli supplementari, 18 e seg. 
fici, finchè tengono l'ufficio $\mathrm{e}$ in seguito al consiglio della parrocchia, od al presidente o sorvegliante della parrocchia, nei distretti rurali e nelle città ai consigli municipali (councils. of the borough, urban districts). Gli equivalenti di decime di beni non compresi nelle suaccennate categorie dovranno devolversi ai Consigli di Contea, e il resto della proprietà devoluta ai Welsh Commissioners passerà poi al Consiglio del Paese di Galles (art. 6 c. d.), fermi restando in tutti questi trasferimenti di proprietà $i$ diritti pubblici e privati ad essi inerenti. I fondi e somme non devoluti alle «rappresentanze» della Chiesa anglicana, secondo le norme suaccennate potranno impiegarsi dai Commissari Gallesi per i seguenti scopi: Erezione o mantenimento di ospedali, dispensarii o istituti per convalescenti; assistenza agli ammalati; fondazione o mantenimento di istituti di cultura o biblioteche; istruzione tecnica o superiore, come pure per scopi di beneficenza o di utile pubblico, a cui non venga provveduto a pubbliche spese (Art. 7 e App. A). É questa una delle clausole più importanti della legge, che sanziona il principio dell' applicazione di beni e redditi ecclesiastici a scopi di beneficenza, di istruzione e di utile pubblico. Alla stessa guisa i redditi devoluti al Consiglio del Paese di Galles, dedotte le spese per l'applicazione della legge, dovranno impiegarsi a promuovere l'istruzione tecnica e superiore. ${ }^{1}$ ) I progetti per queste erogazioni, in cui si dovrà"tener conto dei bisogni specifici delle parocchie ecclesiastiche in cui $\mathrm{i}$ beni sono situati, o da cui derivano i redditi dovranno venir approvati dal Parlamento (Art. 7, n. 2-4). Una serie di disposizioni si riferisce ai compensi dovuti agli attuali investiti di benefici e uffici nella Chiesa anglicana. Tutti gli aventi diritti ad un equivalente di decime (Tithe rent charge), ricevono finchè tengono il relativo ufficio ecclesiastico, una somma annuale corrispondente alla media di sette anni di detto equivalente, dedotto l'importo per l'esazione da deter-

1) Un calcolo approssimativo delle somme incamerate, dopo che verranno estinti gli «interessi esistenti» trovasi in Fowell-Dibdin, o. c. p. $83 \mathrm{seg}$. Secondo questo computo, che é espressamente indicato come incompleto, lo Stato assorbirebbe un reddito annuo di $£$ st. 244,680 , mentre alla Chiesa anglicana gallese resterebbero $£$ st. 19.882 annue. 
La separazione della Stato dalla Chiesa nel Paese di Galles. 275

minarsi dai Commissarii gallesi. ${ }^{1}$ ) Tutti coloro che tengono un ufficio ecclesiastico della Chiesa anglicana, possono (art. 15), col consenso della rappresentanza, entro cinque anni dall' approvazione della legge, permutare $\mathrm{i}$ loro interessi esistenti negli emolumenti dell' ufficio, in una rendita vitalizia da computarsi secondo norme determinate. I patroni di benefici colpiti dalla legge (art. 16), potranno avere, su apposita domanda, cinque anni dopo la separazione, o quando si verifichi una vacanza prima di detto termine, una somma corrispondente ad un' annata degli emolumenti beneficiarii, calcolata sulla media dei tre anni antecedenti all'approvazione della legge (art. 16), esclusi però i benefici regi e quelli appartenenti a corporazioni disciolte dalla legge, o ad enti pubblici o fiduciarii (Trustees). Ai titolari di uffici laici (art. 17), come i cancellieri delle diocesi, i registrars, parish clerks e sacrestani, viene assicurato un congruo compenso da determinarsi dal Tesoro. Da ultimo il progetto contiene norme amministrative per i fondi degli Ecclesiastical Commissioners e del Queen Anne's Bounty, per le fondazioni, per gli oneri dei beneficiati, per la vendita e amministrazione di beni passati ai Commissarii Gallesi o al Consiglio del Paese di Galles, per i registri ecclesiastici, ecc. ed una interpretazione autentica dei termini tecnici usati dalla legge.

Tale nelle linee fondamentali il nuovo progetto di separazione fra lo Stato e la Chiesa nel Paese di Galles, le cui sorti sono intimamente congiunte con quelle del Ministero Asquith. L'approvazione del Parliamentary Bill dell' agosto 1911, che ha limitato il diritto di veto della House of Lords, ha certamente spianata la via all' attuazione legislativa della separazione gallese e si dovrà in seguito constatare se e in quanto essa sia destinata modificare nell'avvenire le tradizionali relazioni fra lo Stato e la Chiesa anglicana in Inghilterra.

1) Il Tithe Commutation Act del 1836 commutava l'importo delle decime in un equivalente in danaro (Rent Charge) corrispondente al prezzo del grano secondo la media di sette anni. Questo principio veniva mantenuto anche dalla susseguente legislazione inglese in materia di decime. Cf. Thring, The Tithe Act 1891, London 1891. 